\title{
Extracellular sugar phosphates are assimilated by Streptomyces in a PhoP-dependent manner
}

\author{
Elodie Tenconi $\cdot$ Samuel Jourdan • \\ Patrick Motte • Marie-Joëlle Virolle • \\ Sébastien Rigali
}

Received: 30 March 2012/ Accepted: 7 June 2012

(C) Springer Science+Business Media B.V. 2012

\begin{abstract}
Filamentous microorganisms of the bacterial genus Streptomyces have a complex life cycle that includes physiological and morphological differentiations. It is now fairly well accepted that lysis of Streptomyces vegetative mycelium induced by programmed cell death $(\mathrm{PCD})$ provides the required nutritive sources for the bacterium to erect sporeforming aerial hyphae. However, little is known regarding cellular compounds released during PCD and the contribution of these molecules to the feeding of surviving cells in order to allow them to reach the late stages of the developmental program. In this work we assessed the effect of extracellular sugar phosphates (that are likely to be released in the environment upon cell lysis) on the differentiation processes.
\end{abstract}

Electronic supplementary material The online version of this article (doi:10.1007/s10482-012-9763-6) contains supplementary material, which is available to authorized users.

E. Tenconi $\cdot$ S. Jourdan $\cdot$ P. Motte $\cdot$ S. Rigali $(\bowtie)$ Centre for Protein Engineering (CIP), Institut de Chimie B6a, University of Liège, 4000 Liège, Belgium e-mail: srigali@ulg.ac.be

P. Motte

Laboratory of Functional Genomics and Plant Molecular Imaging and Centre for Assistance in Technology of Microscopy (CATM), Institute of Botany B22, University of Liège, 4000 Liège, Belgium

\section{M.-J. Virolle}

Institut de Génétique et Microbiologie, Université Paris Sud 11, UMR CNRS 8621, 91405 Orsay Cedex, France
We demonstrated that the supply of phosphorylated sugars, under inorganic phosphate limitation, delays the occurrence of the second round of PCD, blocks streptomycetes life cycle at the vegetative state and inhibits antibiotic production. The mechanism by which sugar phosphates affect development was shown to involve genes of the Pho regulon that are under the positive control of the two component system PhoR/PhoP. Indeed, the inactivation of the response regulator phoP of Streptomyces lividans prevented the 'sugar phosphate effect' whereas the S. lividans ppk (polyphosphate kinase) deletion mutant, known to overexpress the Pho regulon, presented an enhanced response to phosphorylated sugars.

Keywords Sporulation - Antibiotic production . Programmed cell death · Cannibalism · Phosphate limitation

\section{Introduction}

Streptomyces species belong to the soil-dwelling bacterial genus responsible for the production of about two-thirds of useful natural products of microbial origin, including antibiotics (Hopwood 2007). These mycelial bacteria follow a complex developmental program that includes two types of interrelated differentiation i.e., (i) a morphological differentiation where aerial hyphae, whose tips ends will differentiate 
into spores, arise from the substrate (vegetative) mycelium and (ii) a physiological/metabolic differentiation characterized by the production of the so-called secondary metabolites. The onset of both types of differentiation correlates with one of the most sophisticated examples of a programmed cell death (PCD) process in prokaryotes (Granozzi et al. 1990; Miguelez et al. 1999). The latest model suggests two rounds of PCD during Streptomyces life cycle (Manteca et al. 2011). After spore germination, a compartmentalized mycelium grows until it undergoes a first PCD round that is followed by the development of a multinucleated mycelium still devoted to the vegetative life style. The second round of PCD starts when this mycelium produce surfactants-like peptides/proteins necessary for aerial growth (Claessen et al. 2006). This round of PCD is thought to correspond to the short period of growth cessation called the transition phase that takes places during the exponential growth and is followed by a slower regain of growth before entry into stationary phase (Granozzi et al. 1990; Puglia et al. 1995; Manteca et al. 2005). The cell death paroxysm at the transition phase causes intensive release of intracellular compounds into the extracellular environment (Manteca et al. 2006). The latter are thought to serve as nutritive sources for surviving vegetative filaments to grow and differentiate in the aerial structures (Mendez et al. 1985). This form of 'altruistic cannibalism' or 'programmed autophagy' is now commonly admitted but almost nothing is known about the assimilation of the cellular compounds released during PCD (lipids, nucleic acids, proteins, phosphorylated sugars, polyphosphate, ions, etc.). In addition, beyond a nutritive role, the sensing of these cellular compounds by the surviving cells might also constitute a decisive signal for the developmental program.

In this work we demonstrated that, on the specific carbon rich and inorganic phosphate $\left(\mathrm{P}_{\mathrm{i}}\right)$ limited medium R2YE, the exogenous supply of phosphorylated sugars, which are most likely released in the course of the PCD process, leads to a delay in the differentiation process that we called the 'extracellular sugar phosphate (exSP) effect'. In contrast, exogenous addition of their cognate un-phosphorylated sugar had no effect on development whereas the addition of free $P_{i}$ had a similar effect than addition of exSPs suggesting a major contribution of the phosphate moiety. Indeed we demonstrated that the 'exSPs effect' requires the response regulator PhoP that governs the adaptation of the bacteria to $\mathrm{P}_{\mathrm{i}}$ limitation.

\section{Materials and methods}

Bacterial strains, media, and culture conditions

Streptomyces standard techniques were performed as described in (Kieser et al. 2000). Strains were cultivated on the carbon rich and $\mathrm{P}_{\mathrm{i}}$ limited medium solid R2YE medium. Glucose, fructose, glycerol, glucosamine, glucose-6-phosphate, glucose-1-phosphate, fructose-6-phosphate, fructose-1,6-bisphosphate, glucosamine-6-phosphate, and glycerol-3phosphate were purchased from Sigma-Aldrich. $100 \mathrm{Mm} \mathrm{NaH} \mathrm{PO}_{4} / \mathrm{K}_{2} \mathrm{HPO}_{4}$ buffer ( $\mathrm{pH}$ 7.2) was used as source of inorganic phosphate $\left(\mathrm{P}_{\mathrm{i}}\right)$. Streptomyces strains used in this study are listed in Table 1.

Table 1 List of Streptomyces strains used in this study

\begin{tabular}{|c|c|c|}
\hline Streptomyces strains & Genotype & Origin or reference \\
\hline Streptomyces coelicolor M145 & $\begin{array}{l}\mathrm{SCP}^{-}, \mathrm{SCP}^{-}, \\
\text {parental strain }\end{array}$ & Gift John Innes Center (UK) \\
\hline Streptomyces lividans TK24 & & Gift John Innes Center (UK) \\
\hline Streptomyces lividans $\Delta p p k$ & ppk::Shygro & Chouayekh and Virolle (2002) \\
\hline Streptomyces lividans $\Delta$ phoP & phoP::囚aac & Ghorbel et al. (2006) \\
\hline Streptomyces avermitilis & & Gift Leiden Institute of Chemistry (NL) \\
\hline Streptomyces cacaoi DSM 40057 & & $\begin{array}{l}\text { Gift National Defense Research } \\
\text { Institute (S) }\end{array}$ \\
\hline Streptomyces clavuligerus & & Gift Leiden Institute of Chemistry (NL) \\
\hline
\end{tabular}


Scanning electron microscopy

Morphological studies of surface-grown vegetative and aerial hyphae of $S$. coelicolor M145 by cryoscanning electron microscopy were performed as follows. Samples were dehydrated through an ethanol series, critical-point dried and platinum coated $(20 \mathrm{~nm})$ in a Balzers SCD-030 sputter-unit before observation using SEM (JEOL JSM-840A) at $20 \mathrm{kV}$ accelerating voltage (Corbari et al. 2012).

\section{Confocal microscopy}

Surface-grown Streptomyces cultures were processed for microscopy as described previously (Manteca et al. 2005). The permeability assay previously described for Streptomyces was used to stain all samples. Staining of dead and viable cell populations were performed with the cell-impermeant nucleic acid stain (propidium iodide, PI) and with SYTO 9 green fluorescent nucleic acid, respectively, and according to the manufacturer's instructions (LIVE/DEAD BacLight Bacterial Viability Kit, Molecular Probes, L-13 152). Samples were then examined under a Leica TCS-SP5 confocal laser-scanning microscope at a wavelength of 488 and $568 \mathrm{~nm}$ for excitation and $530 \mathrm{~nm}$ (green) or $630 \mathrm{~nm}$ (red) for emission. Images were mixed using the Leica confocal software.

\section{Results}

Effect of extracellular sugar-phosphates on Streptomyces development

We assessed the possibility that exSPs could be used as nutrients by supplying exponentially growing vegetative mycelia of $S$. coelicolor M145 and S. lividans TK24 with glucose-6-phosphate (Glc-6-P), glycerol-3phosphate (Gly-3-P), and their non-phosphorylated forms i.e., glucose (Glc) and glycerol (Gly). As shown in Fig. 1a, Gly and Glc had no effect on development when added after $20 \mathrm{~h}$ of growth to the carbon rich and phosphate limited medium, R2YE. In contrast, Glc-6$\mathrm{P}$, Gly-3-P as well as $\mathrm{P}_{\mathrm{i}}$ retarded the timing of production of pigmented antibiotics prodiginines (Red) and actinorhodin (Act) (Fig. 1a). The inhibition of antibiotic production in S. lividans correlated with a strong delay of sporulation when observed at latter time points (Fig. 1b). Similar developmental delays were observed with other Streptomyces spp. (S. avermitilis, S. clavuligerus, and S. cacaoi; Fig. 1b) in the presence of all exSPs tested (data not shown).

Scanning electron microscopy after $40 \mathrm{~h}$ of growth on R2YE confirmed that the delay in S. coelicolor pigmented antibiotic production (physiological differentiation), induced by exSPs, is also accompanied by a delay in morphological differentiation. Indeed, filaments in contact with Fru-1,6-BP are still at the vegetative state (top part of Fig. 2, zone 3) whereas filaments out of influence of the exSP or in contact with fructose already switched to the aerial life style with their hyphal tips pointing vertically towards the surface of the plate (middle part of Fig. 2, zones 1, 2, and 4). Consistently, staining viable or dying filaments of $S$. coelicolor revealed that only the zone under the influence of Fru-1,6-BP still showed dense accumulation of viable cells at the culture surface while other zones of the plates presented almost exclusively dying cells (bottom part of Fig. 2). These observations indicate a clear delay in the onset of the second round of PCD in hyphae influenced by Fru-1,6-BP. Similar retardation of the occurrence of the second round of PCD was observed in S. clavuligerus (supplementary Fig. S2) and S. lividans (data not shown) using Gly-3-P as exSP source. These results suggest that phosphate limitation is a main trigger factor of the PCD-mediated developmental process on this $\mathrm{P}_{\mathrm{i}}$ limited medium.

Interestingly, the model strain S. coelicolor M145 showed, as other species tested, a delay in development after 44 and $64 \mathrm{~h}$ of incubation (upper part of Fig. 2 and Fig. 1a, respectively), whereas it presented an accelerated onset of sporulation after $96 \mathrm{~h}$ of incubation (Fig. 1b). This peculiar behaviour of S. coelicolor was confirmed by further tests with glucosamine-6-phosphate, fructose-6-phosphate (Fru6-P) and fructose-1,6-bisphosphate (Fru-1,6-BP) (Supplementary Fig. S1). This intriguing 'biphasic' (delayed then accelerated development) will be discussed later on (see "Discussion" section).

The utilization of sugar phosphates is PhoPdependent

Theoretically, exSPs could be up-taken as such by a specific transporter similar to the uhp (uptake of hexose phosphate) system of enteroenvasive Escherichia coli and Salmonella enterica (Götz and Göbel 2010). 


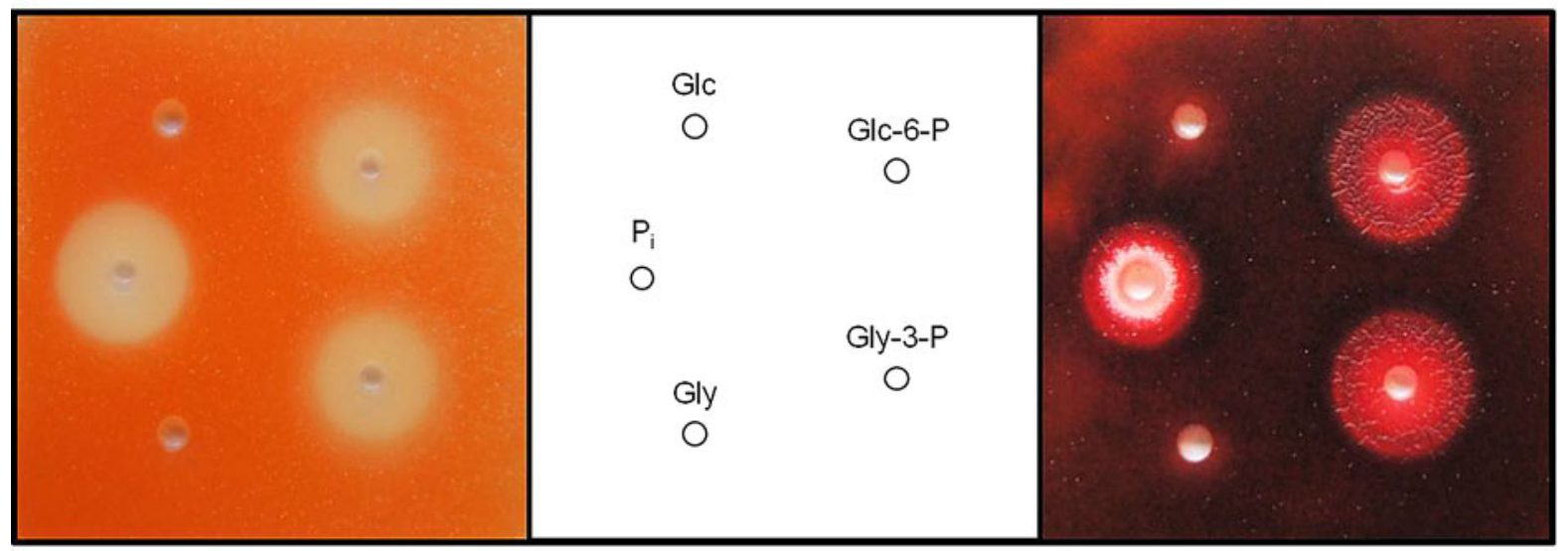

b

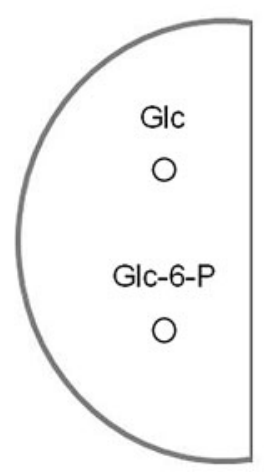

S. avermitilis

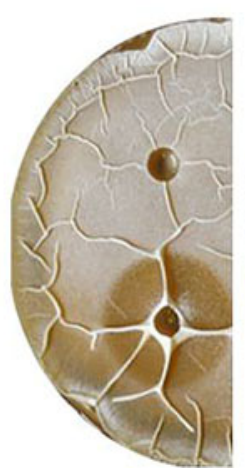

S. cacaoi

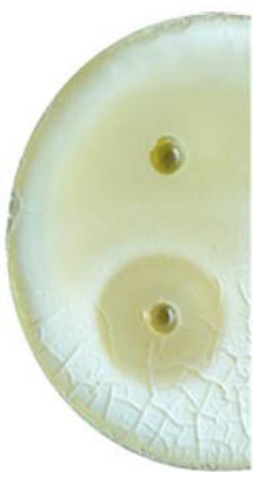

Fig. 1 Sugar phosphates delay development in Streptomyces. a $10^{7}$ spores of $S$. lividans TK24 and S. coelicolor M145 were plated on R2YE solid medium. After $\sim 20 \mathrm{~h}$ of growth, wells ( $5 \mathrm{~mm}$ diameter) were made into the agar and $100 \mathrm{mM}$ of glycerol (Gly), glucose (Glc), glycerol-3-phosphate (Gly-3-P), glucose-6-phosphate (Glc-6-P), and inorganic phosphate $\left(\mathrm{P}_{\mathrm{i}}\right)$ were deposited into them. Pictures were taken after $64 \mathrm{~h}$ of

Alternatively the sugar phosphates can be dephosphorylated by extracellular phosphatases, the carbon moiety being taken-up by the usual transport system of the cognate sugar and the $\mathrm{P}_{\mathrm{i}}$ moiety by known low or high affinity phosphate uptake systems (PitH1 and PitH2, or PstSCAB, (Esteban et al. 2008; Santos-Beneit et al. 2008). Since all the un-phosphorylated carbon sources tested had no impact on development whereas exogenous supply of $\mathrm{P}_{\mathrm{i}}$ led to a developmental delay similar (although not identical) to that observed with exSPs (Fig. 1a), we postulated that the effect of exSPs was mostly due to their P moiety. Indeed, the exSPs effect extends to a wider area when $S$. coelicolor was subjected to exogenous supply of di-phosphorylated

S. clavuligerus

S. lividans

S. coelicolor
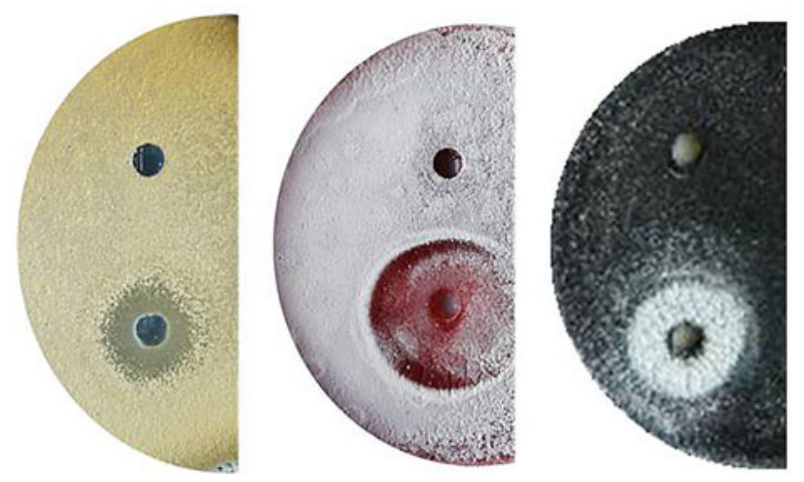

incubation at $28^{\circ} \mathrm{C}$. Note the retarded Red production in S. lividans and Act production in S. coelicolor. b Glc-6-P blocks the onset of sporulation in all Streptomyces species tested while it accelerates aerial hyphae formation in S. coelicolor M145 after $96 \mathrm{~h}$ of incubation at $28{ }^{\circ} \mathrm{C}$ ). The exogenous supply $100 \mathrm{mM}$ of Glc (top well) and Glc-6-P (bottom well) was made as described above

fructose compared to its mono-phosphorylated form (Supplementary Fig. S1) suggesting a dose effect of phosphate.

In order to test this hypothesis, the effect of exogenous supply of exSPs and of $\mathrm{P}_{\mathrm{i}}$ on development was assessed in the phoP mutant of S. lividans. PhoP is the transcriptional response regulator of the two component system (TCS) phoR/phoP that governs the adaptation of the bacteria to a limitation in $\mathrm{P}_{\mathrm{i}}$. Upon $\mathrm{P}_{\mathrm{i}}$ limitation the kinase PhoR autophosphorylates and then transfers its phosphate to the response regulator PhoP. Phosphorylated PhoP is then able to drive the expression of genes of the very large pho regulon which includes, amongst others, all genes 


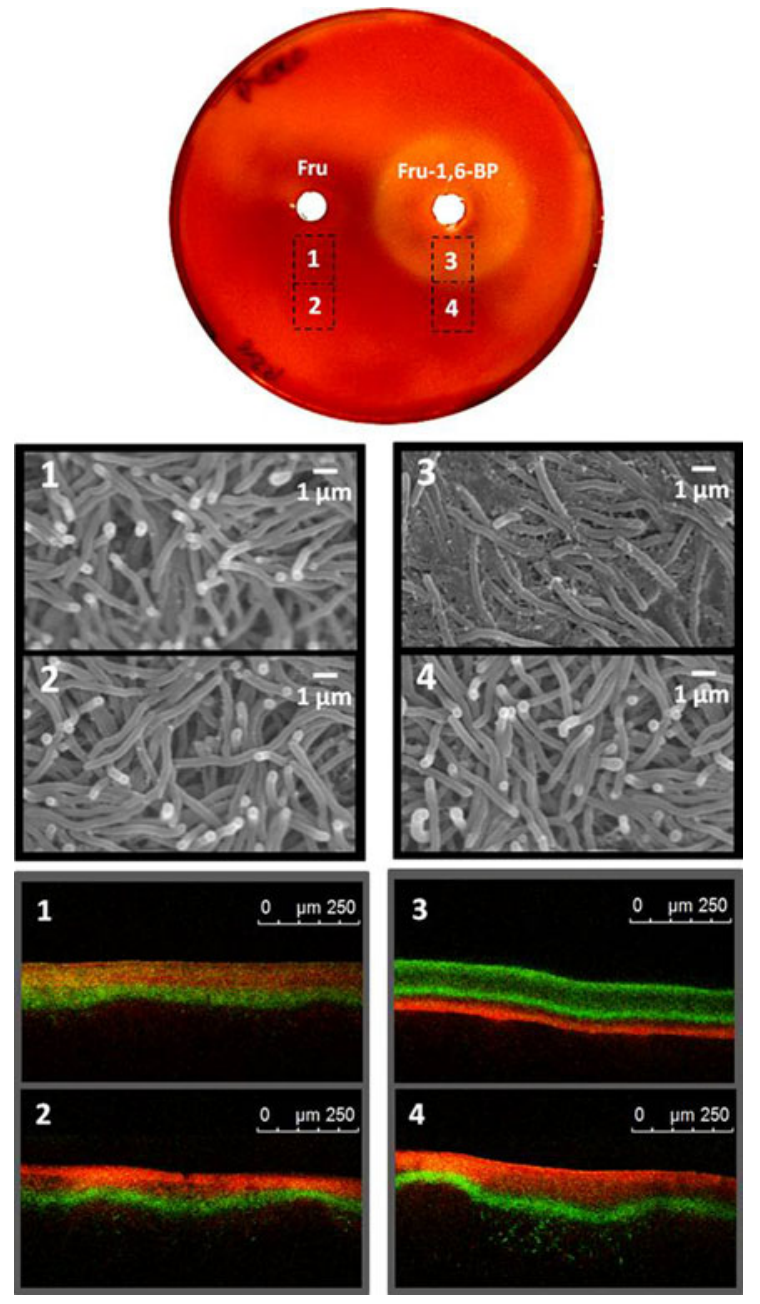

Fig. 2 Sugar phosphates delay the onset of aerial hyphae and the second round of PCD. S. coelicolor M145 was plated on R2YE and exposed to fructose (Fru) or fructose-1,6-biphosphate (Fru-1,6-BP) as described in Fig. 1. The picture on the top was taken after $44 \mathrm{~h}$ of growth (transition phase). Note the reduced production of Red around the well containing the exSP. Zones 1, 2, 3, and 4 were analyzed by cryo-scanning electron microscopy (middle part). Note the absence of aerial hyphal tips within the exSP diffusion area (zone 3). Thin slices of mycelium were cut from the plate and stained with the LIVE/DEAD Bac-Light Bacterial Viability Kit (Invitrogen). Samples were observed under a Leica TCS SP5 II confocal laser-scanning microscope at wavelengths of 488 and $568 \mathrm{~nm}$ for excitation and $530 \mathrm{~nm}$ (green, living cells) or $630 \mathrm{~nm}$ (red, dying cells) for emission (bottom part). Note that the surface of the bacterial culture treated with Fru-1,6BP (zone 3) essentially contains viable cells, while dying cells are mostly present at the surface of zones treated with fructose (zone 1) or outside the zone influenced by the exogenous supplied compounds (zones 2 and 4 ) required for phosphate sources scavenging, uptake, utilisation and storage (Sola-Landa et al. 2003; SolaLanda et al. 2005). This TCS was shown to be highly and constitutively expressed on the carbon rich and $\mathrm{P}_{\mathrm{i}}$ limited medium (R2YE) used in this study (Darbon et al. 2012).

We observe that the exogenous addition of $\mathrm{P}_{\mathrm{i}}$ was still able to block antibiotic production in the S. lividans $\triangle p h o P$ mutant (Fig. 3a) whereas exogenous supply of Glc-1-P or Gly-3-P was not able to inhibit antibiotic (prodiginines) production anymore in that background (Fig. 3a, b). These observations suggest that $\mathrm{P}_{\mathrm{i}}$ is likely to be transported by the PhoP independent PitH1 system (Santos-Beneit et al. 2008) (Fig. 4) whereas exSPs utilisation involves genes of the Pho regulon under the positive control of PhoP. Interestingly, the polyphosphate kinase $p p k$ mutant strain of S. lividans, in which the TCS PhoR/PhoP (and thus genes of the PhoP regulon) was shown to be over-expressed (Ghorbel et al. 2006), displays higher sensitivity to the exSPs than the parental strain (Fig. 3b).

\section{Discussion}

Numerous reports in the literature mention morphogenic molecules that are specifically involved in signalling networks governing metabolite production as well as aerial hyphae and spore formation in Streptomyces (Willey and Gaskell 2011). Although the role of ubiquist molecules in signalisation has been poorly investigated, several studies confirmed that these molecules are likely to signal specific metabolic state and thus also play important regulatory roles (Viollier et al. 2001a, b; Li et al. 2008; Rigali et al. 2008; Luti and Mavituna 2011). For instance, we have previously demonstrated that the peptidoglycan aminosugar building block $N$-acetylglucosamine (GlcNAc) has a different impact on development depending on the nutritional context and its concentration (Rigali et al. 2006, 2008; Nothaft et al. 2010; Craig et al. 2012; Swiatek et al. 2012).

Besides our studies on compounds emanating from the cell wall, we investigated here the possible role in development of cytoplasmic phosphorylated sugars spilled into the extracellular environment. We demonstrated the existence of a general mechanism that 
Fig. 3 Sugar phosphates modulate the onset of development in a PhoPdependent manner. a Spores of S. lividans TK24 (parental strain) and $\Delta p h o P$, were streaked on R2YE solid medium and the following addition of $100 \mathrm{mM}$ of glycerol-3phosphate (Gly-3-P, top left well), glucose-1-phosphate (Glc-1-P, top right well) or inorganic phosphate $(\mathrm{Pi}$, bottom wells) were made as described in the legend of Fig. 1. Pictures were taken after $48 \mathrm{~h}$ of incubation at $28{ }^{\circ} \mathrm{C}$. Note that inactivation of $p h o P$ prevents the 'exSPs effect' but not that of $\mathrm{P}_{\mathrm{i}}$ on development. b The inactivation of the polyphosphate kinase encoding gene $p p k$ renders $S$. lividans more sensitive to the exSP

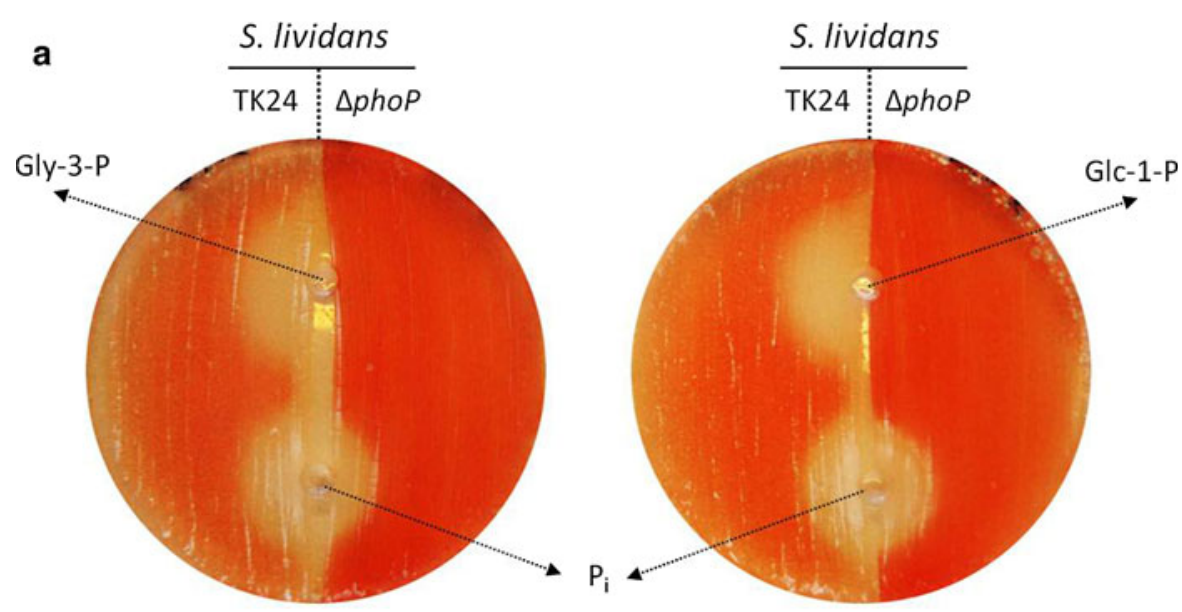

b

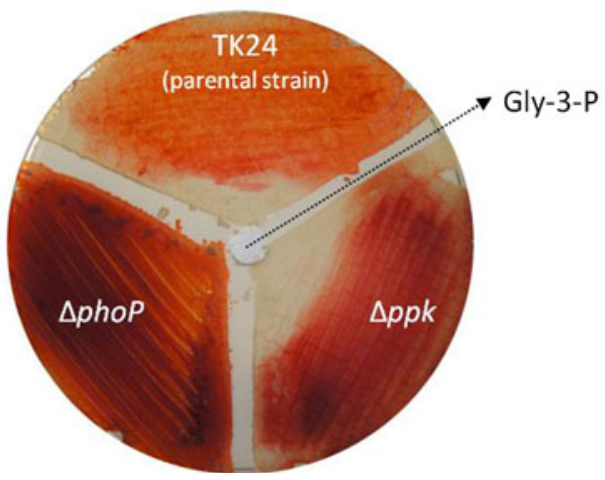

allows the utilization of sugar phosphates as nutrient leading to a delay in the timing of Streptomyces development (PCD, antibiotic production and sporulation). In the R2YE medium limited in $\mathrm{P}_{\mathrm{i}}$, where the expression of $p h o R / p h o P$ was shown to be very high and constitutive (Darbon et al. 2012), the deletion of phoP in S. lividans suppresses the 'exSPs effect' on development but not that of exogenous addition of $\mathrm{P}_{\mathrm{i}}$. In order to rationalize these observations, we propose that the exSPs would be first dephosphorylated by PhoP-dependent secreted phosphatases (such as PhoA and PhoD) (Apel et al. 2007) and the resulting inorganic phosphate would enter the cell by classical medium or high affinity phosphate transporters (PitH2 or PstSCAB; Fig. 4).

However, at this stage of the work, we cannot totally exclude the possibility that exSPs would directly enter the cell via specific transporters similar to the uhp system (Götz and Göbel 2010) and controlled by PhoP. This hypothesis seems unlikely since a genomic scale analysis of possible carbohydrate transporters (Bertram et al. 2004) did not identified, in S. coelicolor, an uhp-like uptake system. Alternatively, exSPs might be directly sensed by a specific still unknown sensory kinase that would also be able to activate PhoP (see Fig. 4 that summarizes the hypothetical ways exSPs could be sensed, uptaken and assimilated in streptomycetes.)

Interestingly, besides their nutritional function at high concentration, some of our results suggest that, at low concentration, the exSP might act as signalling molecules. The sensing of low level of such cellular compounds by the surviving cells might constitute, at the beginning of the second round of PCD, an inducing signal to trigger a cannibalistic behavior in those surviving cells and induce secondary metabolite production and sporulation (Claverys and Havarstein 2007; Gonzalez-Pastor 2011). Indeed, a sporulation ring is observed around the zone of delayed development of lawns of S. coelicolor and S. lividans under the influence of the exSP (zone 2 in Fig. 5). This observation suggests a dual 'nutrient/signal' function of exSPs. At a distance from the wells, where the concentration of the exSP is likely to be low and thus 


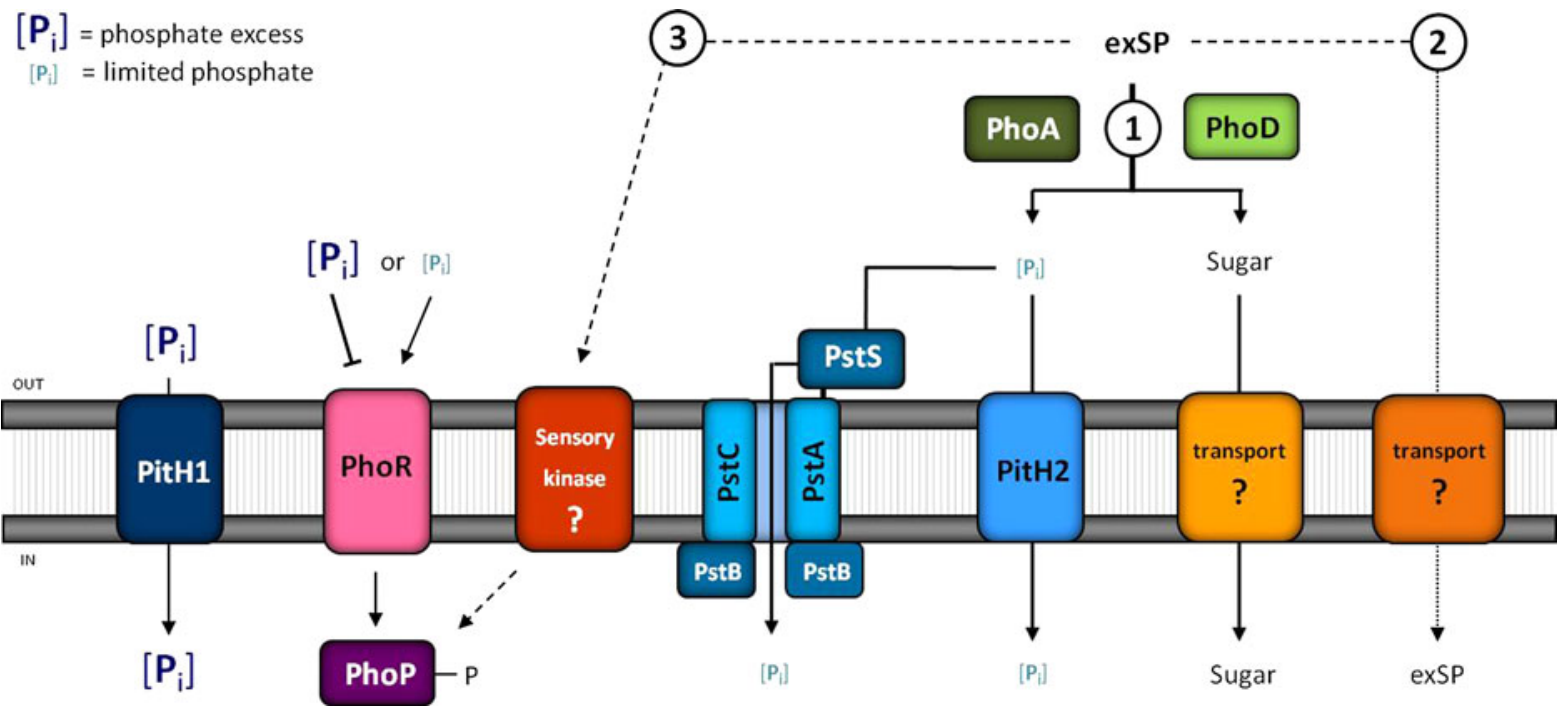

Fig. 4 Favored model (continuous lines, (1) of PhoP-dependant sugar phosphate assimilation. On R2YE, the two components system $p h o R / p h o P$ and thus genes of the Pho regulon under its control, including secreted phosphatases (such as PhoA and PhoD), are highly and constitutively expressed. The secreted phosphatases would dephosphorylate exSPs and the released phosphate would be transported via the PhoP dependent medium and high affinity transporter PitH2 and PstSABC, respectively. Alternative model of putative PhoP-dependant direct sugar phosphate uptake (doted lines, (2)): A direct transport into the cell of exSPs via a putative specific sugarphosphates uptake system controlled by PhoP. Alternative model of putative sugar phosphate sensing (dashed lines, (3)): The exSPs might be sensed by an unknown sensory kinase able to phosphorylate PhoP. $P_{i}$, inorganic phosphate, PhoR phosphate limitation two-component histidine kinase, PhoP phosphate limitation response regulator associated to PhoR, PitH1 low-affinity $P_{i}$ transporter under inorganic phosphate excess, PitH2 $\mathrm{P}_{\mathrm{i}}$ low-affinity transporter under phosphate limitation conditions, PstABC $\mathrm{P}_{\mathrm{i}}$ high-affinity ABC-transporter. The model is deduced from the compilation of previous works (Sola-Landa et al. 2003, 2005; Diaz et al. 2005; Ghorbel et al. 2006; Apel et al. 2007; Esteban et al. 2008; Santos-Beneit et al. 2008)

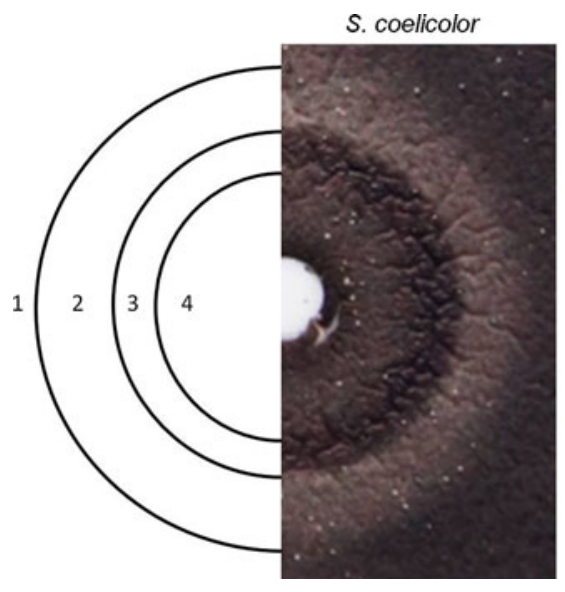

Fig. 5 Concentration dependence of the sugar-phosphates effect on Streptomyces development. Close up on the well of Fig. 1 with exogenous supply of Gly-3-P but taken after $72 \mathrm{~h}$ of incubation at $28{ }^{\circ} \mathrm{C}$. In the plates, a gradient of exSP concentration is established around the wells, with the highest concentration around the wells (zone 4) and the lowest concentration at a distance from the wells in zone 1. In

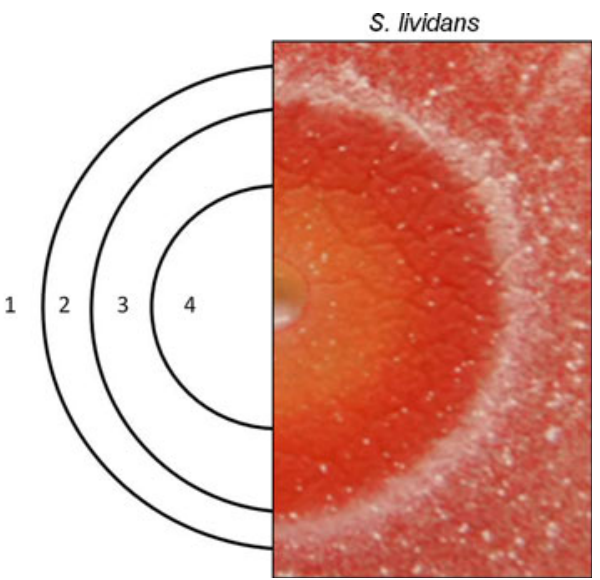

S. coelicolor, Gly-3-P has moderate enhancing effect on sporulation in zone 4, inhibits sporulation in zone 3, and accelerates sporulation in zone 2. In S. lividans, Gly-3-P inhibits Red production and sporulation in zone 4, inhibits sporulation but not Red production in zone 3 , and accelerates sporulation in zone 2 
when the nutritious effect is negligible, exSPs might signal the occurrence of an intensive autolysis linked to PCD that would inform Streptomyces that it is time to start its differentiation program. These observations are reminiscent from that made by Li and collaborators that have previously reported a similar effect of extracellular ATP on the physiology of S. coelicolor (Li et al. 2008). Furthermore, this effect might be related to the peculiar behaviour observed for S. coelicolor (Fig. 1b). Indeed, the accelerated onset of sporulation after $96 \mathrm{~h}$ of incubation might indicate that this strain assimilates exSPs at a higher rate than S. lividans and the other Streptomyces strains, reaching more quickly than the latter the critical low concentration of exSPs that triggers the developmental process.

Studies on the concentration-dependent effect of exSPs on development and on the molecular mechanisms by which exSPs might signal a metabolic/ physiological state are currently under investigation.

Acknowledgments We are thankful to Juan Francisco Martin, and Angel Manteca for interesting discussions. SR is a FRSFNRS research associate. This work is supported by a FRIA fellowship from the FRS-FNRS to ET, by the 'Fonds Spéciaux du Conseil de la Recherche' from the University of Liège to PM and SR (grant 506 R.CFRA.1237), and by FRS-FNRS grants (Grant Nos. 2.4638.05, 2.4540.06, 2.4531.09, 2.4583.08 and 2.4581.10) to PM.

\section{References}

Apel AK, Sola-Landa A et al (2007) Phosphate control of phoA, phoC and phoD gene expression in Streptomyces coelicolor reveals significant differences in binding of PhoP to their promoter regions. Microbiology 153(Pt 10):35273537

Bertram R, Rigali S et al (2011) Regulon of the N-acetylglucosamine utilization regulator NagR in Bacillus subtilis $\mathrm{J}$ Bacteriol 193(14):3525-3536

Chouayekh H, Virolle MJ (2002) The polyphosphate kinase plays a negative role in the control of antibiotic production in Streptomyces lividans. Mol Microbiol 43(4):919-930

Claessen D, de Jong W et al (2006) Regulation of Streptomyces development: reach for the sky! Trends Microbiol 14(7):313-319

Claverys JP, Havarstein LS (2007) Cannibalism and fratricide: mechanisms and raisons d'etre. Nat Rev Microbiol 5(3):219-229

Corbari L, Durand L et al (2012) New digestive symbiosis in the hydrothermal vent amphipoda Ventiella sulfuris. C R Biol 335(2):142-154
Craig M, Lambert S et al (2012) Unsuspected control of siderophore production by $\mathrm{N}$-acetylglucosamine in Streptomycetes. Environ Microbiol Rep. doi:10.1111/j1758222920 $1200354 \mathrm{x}$

Darbon E, Martel C, et al. (2012) Transcriptional and preliminary functional analysis of the six genes located in divergence of phoR/phoP in Streptomyces lividans. Appl Microbiol Biotechnol. doi:10.1007/s00253-012-3995-2

Diaz M, Esteban A et al (2005) The high-affinity phosphatebinding protein PstS is accumulated under high fructose concentrations and mutation of the corresponding gene affects differentiation in Streptomyces lividans. Microbiology 151(Pt 8):2583-2592

Esteban A, Diaz M et al (2008) Expression of the $p s t S$ gene of Streptomyces lividans is regulated by the carbon source and is partially independent of the PhoP regulator. BMC Microbiol 8:201

Ghorbel S, Kormanec J et al (2006) Transcriptional studies and regulatory interactions between the phoR-phoP operon and the phoU, mtpA, and ppk genes of Streptomyces lividans TK24. J Bacteriol 188(2):677-686

Gonzalez-Pastor JE (2011) Cannibalism: a social behavior in sporulating Bacillus subtilis. FEMS Microbiol Rev 35(3):415-424

Götz A, Göbel W (2010) Glucose and glucose 6-phosphate as carbon sources in extra- and intracellular growth of enteroinvasive Escherichia coli and Salmonella enterica. Microbiology 156(Pt 4):1176-1187

Granozzi C, Billetta R et al (1990) A breakdown in macromolecular synthesis preceding differentiation in Streptomyces coelicolor A3(2). J Gen Microbiol 136(4):713-716

Hopwood D (2007) Streptomyces in nature and medicine: the antibiotic makers. Oxford University Press, New York

Kieser T, Bibb MJ et al (2000) Practical streptomyces genetics. John Innes Foundation, Norwich

Li M, Kim TJ et al (2008) Effects of extracellular ATP on the physiology of Streptomyces coelicolor A3(2). FEMS Microbiol Lett 286(1):24-31

Luti KJ, Mavituna F (2011) Elicitation of Streptomyces coelicolor with dead cells of Bacillus subtilis and Staphylococcus aureus in a bioreactor increases production of undecylprodigiosin. Appl Microbiol Biotechnol 90(2): 461-466

Manteca A, Fernandez M et al (2005) Mycelium development in Streptomyces antibioticus ATCC11891 occurs in an orderly pattern which determines multiphase growth curves. BMC Microbiol 5:51

Manteca A, Fernandez M et al (2006) Cytological and biochemical evidence for an early cell dismantling event in surface cultures of Streptomyces antibioticus. Res Microbiol 157(2):143-152

Manteca A, Ye J et al (2011) Phosphoproteome analysis of streptomyces development reveals extensive protein phosphorylation accompanying bacterial differentiation. J Proteome Res 10(12):5481-5492

Mendez C, Brana AF et al (1985) Role of substrate mycelium in colony development in Streptomyces. Can J Microbiol 31(5):446-450

Miguelez EM, Hardisson C et al (1999) Hyphal death during colony development in Streptomyces antibioticus: morphological evidence for the existence of a process of cell 
deletion in a multicellular prokaryote. J Cell Biol 145(3):515-525

Nothaft H, Rigali S et al (2010) The permease gene nagE2 is the key to $\mathrm{N}$-acetylglucosamine sensing and utilization in Streptomyces coelicolor and is subject to multi-level control. Mol Microbiol 75(5):1133-1144

Puglia AM, Vohradsky J et al (1995) Developmental control of the heat-shock stress regulon in Streptomyces coelicolor. Mol Microbiol 17(4):737-746

Rigali S, Nothaft H et al (2006) The sugar phosphotransferase system of Streptomyces coelicolor is regulated by the GntR-family regulator DasR and links $N$-acetylglucosamine metabolism to the control of development. Mol Microbiol 61(5):1237-1251

Rigali S, Titgemeyer F et al (2008) Feast or famine: the global regulator DasR links nutrient stress to antibiotic production by Streptomyces. EMBO Rep 9(7):670-675

Santos-Beneit F, Rodriguez-Garcia A et al (2008) Phosphatedependent regulation of the low- and high-affinity transport systems in the model actinomycete Streptomyces coelicolor. Microbiology 154(Pt 8):2356-2370
Sola-Landa A, Moura RS et al (2003) The two-component PhoR-PhoP system controls both primary metabolism and secondary metabolite biosynthesis in Streptomyces lividans. Proc Natl Acad Sci USA 100(10):6133-6138

Sola-Landa A, Rodriguez-Garcia A et al (2005) Binding of PhoP to promoters of phosphate-regulated genes in Streptomyces coelicolor: identification of PHO boxes. Mol Microbiol 56(5):1373-1385

Swiatek MA, Tenconi E et al (2012) Functional analysis of the $\mathrm{N}$-acetylglucosamine metabolic genes of Streptomyces coelicolor and role in control of development and antibiotic production. J Bacteriol 194(5):1136-1144

Viollier PH, Minas W et al (2001a) Role of acid metabolism in Streptomyces coelicolor morphological differentiation and antibiotic biosynthesis. J Bacteriol 183(10):3184-3192

Viollier PH, Nguyen KT et al (2001b) Roles of aconitase in growth, metabolism, and morphological differentiation of Streptomyces coelicolor. J Bacteriol 183(10):3193-3203

Willey JM, Gaskell AA (2011) Morphogenetic signaling molecules of the streptomycetes. Chem Rev 111(1):174-187 